\title{
A hybrid meta-heuristic for the VRPTW with cluster-dependent tour starts in the newspaper industry
}

\author{
Dominik Böhnlein, Christian Gahm, Axel Tuma \\ Department of Production and Environmental Management, University of Augsburg, Germany \\ [dominik.boehnlein |christian.gahm |axel.tuma]@wiwi.uni-augsburg.de
}

\begin{abstract}
The newspaper industry implies a special optimization problem: Vehicle routes have to be executed directly after print finalization in order to supply customers in time. Hereby, the underlying vehicle routing problem with time windows has additional constraints: heterogeneous editions per vehicle and cluster-dependent tour starts due to differing completion times of editions.

In this contribution, this problem is defined as a vehicle routing problem with time windows and cluster-dependent tour starts (VRPTWCD), caused by the allocation of editions to clusters and different edition completion times. This implicates the need for a new solution procedure. The well known methods Ant Colony Optimization and Tabu Search are combined and enhanced by additional parameters, resulting in a first approach (named AntTabu) for solving the VRPTWCD. This hybrid meta-heuristic, integrated in an application software for the newspaper industry, is applied to a real life application case of one of the largest newspaper companies in Germany.
\end{abstract}

\section{Introduction}

In recent years newspaper companies had to face the competition with new media, especially the internet, and in many cases decreasing advertising revenues [29]. The companies react to these challenges with two approaches, by reducing transport costs and by increasing customer satisfaction. The reduction of transport costs can be achieved on the one hand by optimized vehicle routes and on the other hand by using fewer vehicles. The increase of customer satisfaction is more difficult to realize because customers have two competitive requirements: They want to read the latest news and want to get the news in time in the morning. To obtain these requirements it is necessary to analyze the newspaper production and logistics processes.
Figure 1 gives a brief overview of the process flow in the newspaper industry.

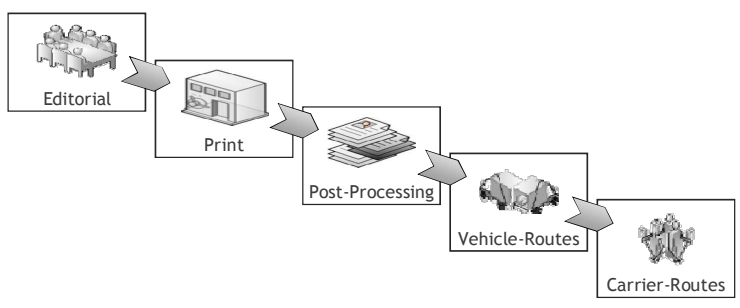

Figure 1. Processes in the newspaper industry

The editorial department creates the content of different editions and defines a new production schedule for the print department each production day. This schedule depends on upcoming news as well as on the print run of each edition. In many cases printing is followed by a post-processing where pre-prints and advertising supplements are inserted. After this step newspapers are ready for delivery. The newspapers are bundled and allocated to trucks which deliver the bundles to unloading points where newspaper carriers are waiting. The newspaper carriers themselves deliver each single newspaper to a customer. The process flow shows the particular complex situation in which production and logistics are directly coupled.

As mentioned before, the aim of getting a high customer satisfaction depends on two aspects: the first is to publish the most recent news, the second to deliver the newspapers in time. Consequently, the editorial deadline has to be as late as possible and the newspaper carriers have to start at a specific deadline to supply customers in time. The production and logistics processes are located between these two points of time, whereas the print and post-processing schedules are predefined by the editorial department and therefore the completion time for each edition is pre-defined and changes each day. The following logistics process of delivering newspapers to the unloading points directly influences the level of 
service. If unloading points are not supplied in time, news carriers are not able to deliver newspapers punctually. But not only customer satisfaction is affected if tardiness occurs: Additional costs emerge if newspaper carriers have to wait for their editions (carrier compensation costs). This could be avoided by optimized vehicle routes. The first objective of minimizing transport costs can also be realized by optimizing the allocation of unloading points to routes (clustering) and by optimizing the vehicle routes (routing).

The objectives of minimizing transport costs and maximizing customer satisfaction as well as the additional conditions stated above define a typical newspaper distribution problem that can be formulated on the basis of a vehicle routing problem with time windows (VRPTW). For a detailed description see [6,7].

\section{VRPTW in the newspaper industry}

The VRPTW in the newspaper industry can briefly be described as the task of delivering several different newspaper editions $e \in\{1, \ldots, \mathrm{E}\}$ from a depot (production site) to unloading points $i \in$ $\{1, \ldots, N\}$. Each unloading point $i$ has a demand for a certain amount of newspapers $d_{i e}$ of one or more different editions and its supply (including service time $s_{i}$ that is needed for unloading all bundles) has to be realized within a specified time window (in this contribution: one sided time windows with predefined due dates $l_{i}$ ). Each unloading point has to be served by exactly one vehicle $k \in\{1, \ldots, \mathrm{K}\}$ (no split delivery) and each vehicle executes exactly one tour (serves one cluster of unloading points). This problem can be modeled through a connected digraph $\mathrm{G}=(V, \mathrm{~A})$ consisting of a set of nodes $V$ (depot and unloading points) and a set of $\operatorname{arcs} A$ with nonnegative weights (transport costs $t c_{i j}$ and travel times $\left.t_{i j}\right)$. Further constraints are the maximal loading capacity $\left(c a p_{k}\right)$ of a vehicle $k$ and the requirement that all vehicles have to return to the depot.

This kind of problem is well discussed in literature and many researchers have dealt with the newspaper delivery problem. One of the first papers that came up with this topic was published by Holt and Watts [20]. They discussed general guidelines for routing and scheduling in the newspaper industry. The first publication including a mathematical formulation was proposed by Mantel and Fontein [23]. The presented model was simplified by an approximation with respect to time constraints and solved by a heuristic. Most approaches dealing with the VRPTW consist of two steps. They are based either on route-first cluster-second or cluster-first route-second methods. The first group of algorithms calculates one optimized TSP and then divides it into feasible routes [1]. The cluster-first route-second methods identify clusters of customers, in the context of this contribution unloading points, and then minimize the costs of each route serving one cluster. Examples for this approach are the multi-start local search algorithm developed by [5] and the recently published contribution [29] presenting a Tabu Search meta-heuristic. The latter one formulated the problem as an open vehicle routing problem with time windows and zoning constraints.

Bräysy et al. [6,7] stated that the vehicle routing problem with time windows is an NP-hard problem and that it requires heuristic solution strategies for most real-life instances. According to their broad overview meta-heuristics as proposed by $[2,3,4$, $21,22,24]$ seem to perform best.

The problem this contribution deals with has one additional aspect which has, to the authors' knowledge, never been considered in the literature before: cluster-dependent tour start times.

\subsection{The VRPTW with cluster-dependent tour starts (VRPTWCD)}

Unlike the standard VRPTW, cluster-dependent tour start times $t s_{k}$ involve the problem that vehicles are not able to start their tours at a predefined point of time. Their tour start times depend on the predefined production schedule and on the required editions of each cluster (route). Hereby, the required editions of a cluster are determined by the demands of the clusters' unloading points.

Figure 2 shows an exemplary post-processing schedule containing 19 editions which is directly executed after printing the newspapers.

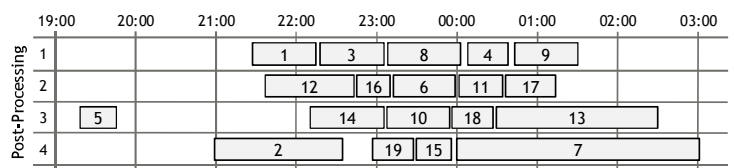

Figure 2. Post-processing schedule

A post-processing schedule defines completion times $f_{e}$ for each edition. Table 1 shows the dependency between cluster requirements (vehicle loads), completion times and start times. 
Table 1. Cluster-dependent tour starts

\begin{tabular}{c|c|c|c}
\hline \multicolumn{2}{c|}{ Vehicle $\mathbf{k}_{\mathbf{1}}$} & \multicolumn{2}{c}{ Vehicle $\mathbf{k}_{\mathbf{2}}$} \\
\hline $\begin{array}{c}\text { cluster } \\
\text { requirements } \\
\text { (edition) }\end{array}$ & $f_{e}$ & $\begin{array}{c}\text { cluster } \\
\text { requirements } \\
\text { (edition) }\end{array}$ & $f_{e}$ \\
\hline 1 & $22: 16$ & 1 & $22: 16$ \\
17 & $01: 18$ & 9 & $01: 38$ \\
\multicolumn{2}{c|}{$t s_{1}=01: 18$} & 12 & $22: 32$ \\
\hline
\end{tabular}

Vehicle $\mathrm{k}_{1}$ requires the editions 1 and 17 which have completion times of $22: 16$ and $01: 18$ respectively. This determines the tour start at 01:18. Vehicle $\mathrm{k}_{2}$ requires the editions 1,9 and 12 , thus the deduced start time is 01:38.

These dependencies can result in the problem illustrated in Figure 3. According to Table 1 the required editions for vehicle $\mathrm{k}_{1}$ are 1 and 17 (unloading point 4 has a demand $d_{4,17}>0$ for edition 17 and unloading point 3 for edition $1-d_{3,1}>0$ ). Vehicle $\mathrm{k}_{2}$ requires the editions 1,9 and $12\left(d_{6,1}>0\right.$, $d_{5,9}>0$ and $d_{7,12}>0$ ).
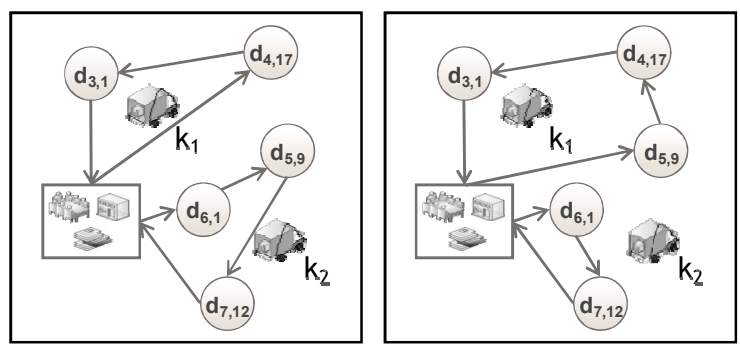

Figure 3. Cluster-dependent start times

The left figure illustrates vehicle $\mathrm{k}_{1}$ delivering editions 1 and 17, thus starting at 01:18, whereas vehicle $\mathrm{k}_{2}$ delivers editions 1,9 and 12 , thus starts at 01:38. Assuming that in this situation all unloading points can be supplied in time and all other constraints are fulfilled, the clusters are called feasible. However, if e.g. unloading point 5 is moved from the cluster served by vehicle $\mathrm{k}_{2}$ to the cluster served by vehicle $\mathrm{k}_{1}$, the start time of vehicle $\mathrm{k}_{1}$ changes to $01: 38$ whereas the start time of vehicle $k_{2}$ changes to $22: 32$. The problem arising with these new start times is that the cluster served by vehicle $\mathrm{k}_{1}$ might not be feasible anymore because of the due dates $\left(l_{3}\right.$ and $\left.l_{4}\right)$ and the delay of start time $t_{1}$ by 20 minutes. This dependency between clusters and start times has to be regarded as an additional constraint during the optimization which includes the allocation of unloading points to clusters (see section 2.2 restrictions (7) and (8)).

\subsection{Mathematical formulation}

The above described VRPTWCD can be formulated mathematically as follows:

Indices:

$\begin{array}{ll}i, j & \text { indices for unloading points } \\ k & \in\{1, \ldots, \mathrm{N}\} ; 0 \text { denotes the depot } \\ e & \text { index for vehicles } \in\{1, \ldots, \mathrm{K}\} \\ & \text { index for editions } \in\{1, \ldots, \mathrm{E}\}\end{array}$

Parameters:

$N \quad$ total number of unloading points

E total number of editions

$t c_{i j} \quad$ transport costs between unloading point $i$

$t c_{i j} \quad$ and unloading point $j$

$t_{i j} \quad$ travel time between unloading point $i$ and

unloading point $j$

$f_{e} \quad$ completion time of edition $e$

$d_{i e} \quad$ demand for edition e at unloading point $i$

(number of copies)

cap capacity of identical vehicles (max.

$w_{e} \quad$ weight per copy of edition $e$

$l_{i} \quad$ due date at unloading point $i$

$s_{i} \quad$ service time at unloading point $i$

$c c \quad$ newspaper carrier compensation costs

(per minute of delay)

$n c_{i}$

number of newspaper carriers at unloading point $i$

Variables:

1 , if there is an arc from unloading point $i$

$x_{i j k} \in\{0,1\} \quad$ to unloading point $j$ supplied by vehicle $k$,

else $0 ; i \neq j$

$t_{i} \quad$ arrival time at unloading point $i$

$t s_{k} \quad$ tour start of vehicle $k$

$t s_{k e} \quad$ tour start of vehicle $k$ concerning edition $e$

$K \quad$ total number of vehicles

Objective function:

$$
\begin{aligned}
\operatorname{Min} \sum_{i=0}^{N} \sum_{j=0}^{N} \sum_{k=1}^{K} t c_{i j} x_{i j k} & \\
& +c c \sum_{i=1}^{N} n c_{i} * \max \left\{\left(t_{i}-l_{i}\right), 0\right\}
\end{aligned}
$$

subject to

$\sum_{j=1}^{N} x_{0 j k}=\sum_{i=1}^{N} x_{i 0 k}=1 \quad \forall k \in\{1, \ldots, \mathrm{K}\}$ 


$$
\begin{array}{lc}
\sum_{k=1}^{K} \sum_{j=0}^{N} x_{i j k}=1 & \forall i \in\{1, \ldots, \mathrm{N}\} \\
\sum_{k=1}^{K} \sum_{i=0}^{N} x_{i j k}=1 & \forall j \in\{1, \ldots, \mathrm{N}\} \\
\sum_{i=0}^{N} \sum_{e=1}^{E} d_{i e} w_{e} \sum_{j=0}^{N} x_{i j k} \leq c a p & \forall k \in\{1, \ldots, \mathrm{K}\} \\
t_{j}=\left\{\begin{array}{rr}
t s_{k}+t_{0 j} & \text { if } x_{0 j k}=1 \\
\sum_{i=1}^{N} x_{i j k}\left(t_{i}+s_{i}+t_{i j}\right) & \text { if } x_{0 j k}=0 \\
\forall j \in\{1, \ldots, \mathrm{N}\}, \forall k \in\{1, \ldots, \mathrm{K}\}
\end{array}\right.
\end{array}
$$

$t s_{k e}= \begin{cases}f_{e} & \text { if } \sum_{i=1}^{N} \sum_{j=1}^{N} d_{i e} x_{i j k}>0 \\ 0 & \text { else }\end{cases}$

$$
\forall k \in\{1, \ldots, \mathrm{K}\}, \forall e \in\{1, \ldots, \mathrm{E}\}
$$

$$
t s_{k}=\max \left\{t s_{k 1}, \ldots, t s_{k E}\right\} \quad \forall k \in\{1, \ldots, \mathrm{K}\}
$$

The non-linear objective function (1) consists of the two challenges as formulated above. The first part minimizes transport costs caused by the vehicle routes. The second part minimizes news carrier compensation costs and thus minimizes the tardiness at the unloading points (implying an increase of customer satisfaction). Altogether the variable logistics costs have to be minimized. In contrast to a standard VRPTW, the number of vehicles in a fleet is predetermined in many newspaper companies and thus the minimization of vehicles is not an objective in this contribution. Equation (2) ensures that each route executed by vehicle $\mathrm{k}$ starts and ends at the depot and that each vehicle leaves the depot only once per day. (3) and (4) specify that each unloading point can be visited only once by one vehicle (no split deliveries; each unloading point has exactly one incoming and one outgoing arc). Capacity constraint (5) ensures that the maximum vehicle capacity is kept. Arrival times are calculated recursively as shown in (6). The tour start of a vehicle concerning an edition is defined in (7). Thus, the tour start of vehicle $\mathrm{k}$ is defined as the latest completion time of all of its demanded editions (8).

Because of the fact that, as shown in [6,7], the VRPTW is a NP-hard problem, the described VRPTWCD is also NP-hard. Consequently heuristic solution strategies are required to solve this real world problem. As newspaper companies need an efficient computation of vehicle routes (time between determination of production schedules and first vehicle routes is limited), computing time is an important claim for an approach solving the VRPTWCD.

\section{Hybrid meta-heuristic AntTabu}

According to the literature, one of the most competitive approaches for a standard VRPTW are the meta-heuristics Ant Colony Optimization (ACO) and Tabu Search (TS) [7]. However, several problems have to be faced in this contribution: First of all, the underlying problem that occurs in the newspaper industry, the VRPTWCD, implicates the need for an extension of those approaches. Secondly, the ACO heuristic can be used for an efficient computation of initial solutions, but needs a local search (as a synonym for neighborhood search) optimization component in order to achieve better solutions [10]. Thirdly, Tabu Search is one of the most competitive heuristics for vehicle routing problems with time windows, but its initial solution has to be chosen in such a way that its generation is fast and easy [28]. E.g. the usage of an initial solution containing tours $[0, i, 0] \forall i \in\{1, \ldots, \mathrm{N}\}$ would lead to poor computing times.

Consequently the need for a new solution approach for the VRPTWCD including the ideas of the most competitive VRPTW approaches, ACO and $\mathrm{TS}$, becomes obvious. Hereby, the current procedures have to be extended by some new ideas in order to face the production schedule- and cluster-dependent tour starts. The resulting meta-heuristic, named AntTabu, uses the advantages of ACO (the efficient computation of initial solutions) and of TS (the efficient local search) and eliminates the disadvantages through an integration of both approaches.

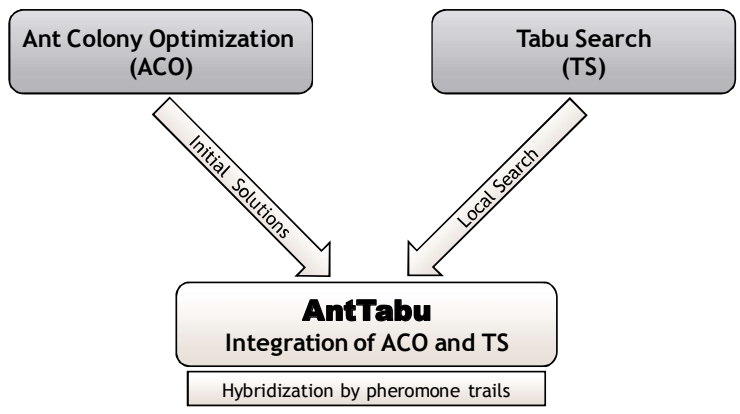

Figure 4. AntTabu - Integration of ACO and TS 


\subsection{AntTabu}

The main AntTabu's course of actions can be stated by the following pseudocode.

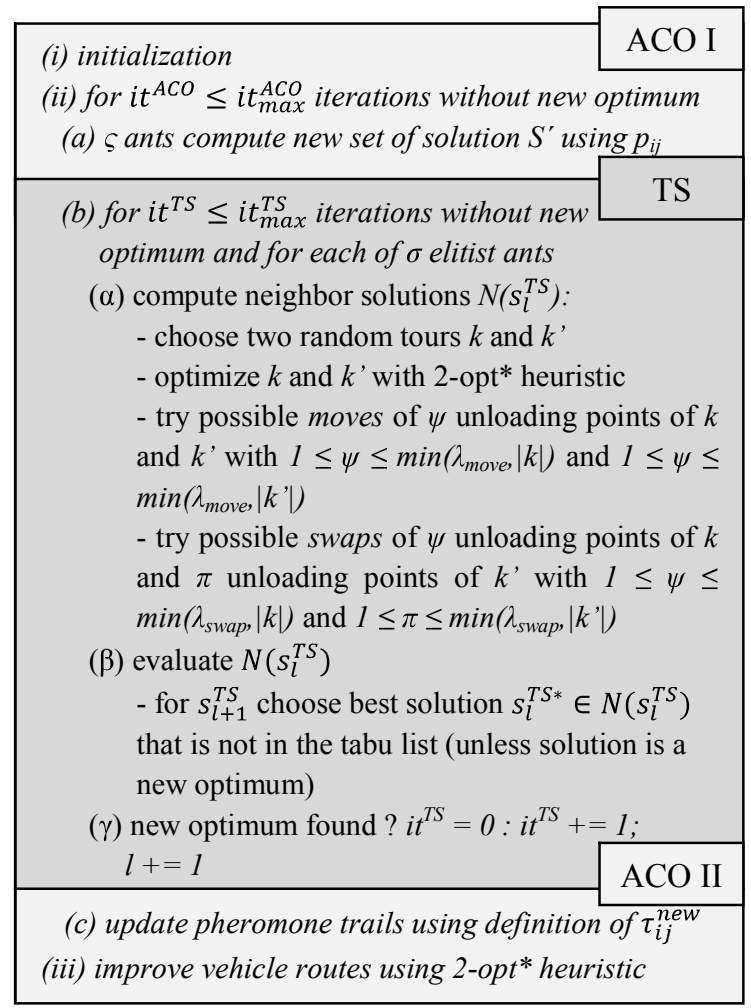

The AntTabu meta-heuristic is composed of two ACO fragments (ACO I + II), enclosing one TS component. These parts can be described as follows:

\section{$\underline{\mathrm{ACO}}$ I}

The first part of AntTabu consists of Ant Colony Optimization, which was originally introduced by Dorigo et al. in the early 1990s [15,16]. Since this introduction, many different ACO implementations arose (e.g. MACS-VRPTW [19], SavingsAnts [14] and DAnts [27]). Reimann et al. presented a unified ant system for four different problem classes [26]. An up-to-date theoretical analysis of ACO is proposed by Dorigo and Blum [18]. The adaptation of e.g. MACSVRPTW (which seems to be an outstanding ACO meta-heuristic according to the literature) to the VRPTWCD is not realizable as MACS-VRPTW focuses on the objectives of minimizing the number of tours and minimizing total travel time (in contrast to this contribution where costs are to be minimized). Hence, the ACO implementation in this work is based on the main course of actions as proposed by $[11,17,19,26]$, as these implementations seem to perform good concerning the VRPTWCD.
AntTabu starts with the initialization of all parameters and variables. For $i t_{\max }^{A C O}$ iterations without new optimum, $\varsigma$ ants generate a new set of solutions $S^{\prime}$ by visiting all unloading points $i \in$ $\{1, \ldots, N\}$ (compare [19]). Each ant starts at a randomly chosen unloading point $i$ and selects an unloading point $j$ according to an exploitation factor $q_{0}$ (as proposed by [19]) as well as to the extended random-proportional rule $p_{i j}$ (originally proposed by [17]). This rule can be stated as follows:

$$
p_{i j}=\left\{\begin{array}{c}
\frac{\left[\tau_{i j}\right]^{\alpha}\left[\eta_{i j}\right]^{\beta}\left[\delta_{i j}\right]^{\gamma}}{\sum_{h \in \Omega}\left[\tau_{i h}\right]^{\alpha}\left[\eta_{i h}\right]^{\beta}\left[\delta_{i h}\right]^{\gamma}} \text { if } j \in \Omega \\
0 \quad \text { otherwise }
\end{array}\right.
$$

whereas $\Omega=\{i \in\{1, \ldots, \mathrm{N}\} \mid i$ is feasible $\} \cup\{0\}$ is a set of unloading points that are feasible to be visited (feasible means that the unloading points have not been visited yet and that due times and capacity restrictions are kept). The random-proportional rule proposed in this contribution consists of three aspects that are taken into account:

- The quality of unloading point $j$ in prior solutions (containing an arc from unloading point $i$ to unloading point $j$ ) - information that is stored in the pheromone trails $\tau_{i j}$ (see description below in ACO II).

- The current quality of unloading point $j$, expressed by the visibility function $\eta_{\mathrm{ij}}$. Researchers propose several definitions for this parameter, e.g. a parametrical savings function [11]. However, concerning the underlying VRPTWCD, the reciprocal of transport costs $\eta_{i j}=\frac{1}{t c_{i j}}$ seems to be the best definition.

- The function $\delta_{\mathrm{ij}}$, indicating if the supply of unloading point $j$ implicates a later tour start $t s_{k}^{\prime}$ in comparison to the current tour start $t s_{k}$ This is expressed by the reciprocal of the tour start postponement (unit: minutes):

$$
\delta_{i j}=\frac{1}{\max \left\{\left(t s_{k}^{\prime}-t s_{k}+1\right), 1\right\}}
$$

Additionally, the proportional rule $p_{i j}$ contains the parameters $\alpha, \beta$ and $\gamma$ that determine the relative influence of pheromone trails, visibility and clusterdependent tour starts. Summarizing, each ant (being at unloading point $i$ ) supplies an unloading point $j$ with a higher possibility, if:

- $\quad j$ led to low costs in prior solutions.

- $j$ causes low transport costs in the current situation. 
- $\quad j$ does not delay the current tour start or merely causes a low tour start delay.

The following figure contains an example for an easier understanding of the extended randomproportional rule for the VRPTWCD.

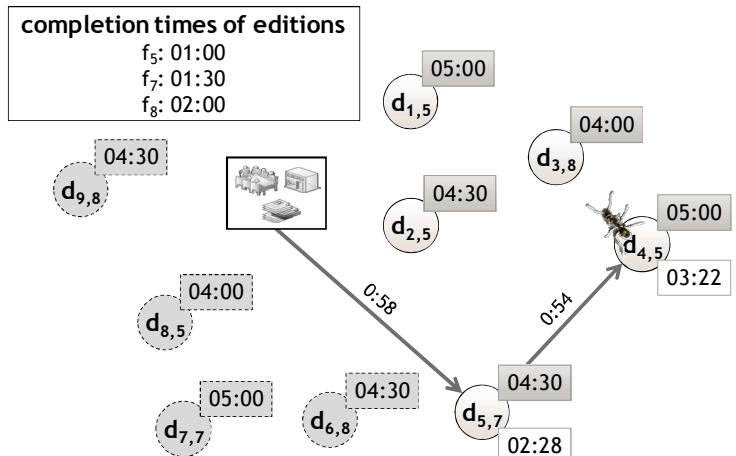

Figure 5. Ant Colony Optimization

Assuming that ant $k$ started its tour at unloading point 5 and decided to use the arc $(5,4)$, it currently supplies unloading point 4 . The current arrival time can be computed by backward calculation. As unloading points 4 and 5 have demands for editions 5 and $7, t s_{k}=\max \left\{\ldots, f_{5}=01: 00, \ldots, f_{7}=01: 30, \ldots\right\}=$ 01:30. Consequently, the arrival time of unloading point 4 is 03:22 $(01: 30+0: 58+0: 54)$. Considering the fact that the unloading points with dotted borders are not feasible anymore $(\notin \Omega)$, the ant now chooses between the unloading points $0,1,2$ and 3 according to $p_{i j}$. This choice depends on prior uses of the arcs $(4,0),(4,1),(4,2)$ and $(4,3)$, i.e. the pheromone trails $\tau_{i j}$, the visibility function $\eta_{i j}$ (unloading point 3 is chosen with the highest possibility because of its low distance and thus low transport costs) and the tour start postponement $\delta_{i j}$. If unloading point 3 was chosen, $t s_{k}$ would be postponed by 30 minutes $\left(t s_{k}=\right.$ $f_{8}=02: 00$ ). Thus, the lower $\delta_{43}$ decreases the (in comparison to the other unloading points) higher $\eta_{43}$.

After $\varsigma$ ants supplied all unloading points $i \in\{1, \ldots, \mathrm{N}\}$ and returned to the depot, the $\sigma$ best ants (so called elitist ants) have the possibility to improve their suboptimal clusters with Tabu Search. The resulting clusters of the non-elitist ants are discarded as they are not allowed to participate in the pheromone trail update because their objective value is too poor (see ACO II below).

\section{TS}

Each of the $\sigma$ elitist ants executes a Tabu Search (the main course of actions is implemented according to the literature $[12,25,31]$ ) that is adapted to the
VRPTWCD and extended by some new ideas. The objective of TS is the optimization of the elitist ants' suboptimal vehicle routes by routing and clustering operations. Hereby, the initial solution consists of the elitist ants' tours. For $i t_{\max }^{T S}$ iterations without new optimum, TS calculates a new neighborhood for the current best solution $s_{l}^{T S}$ : First of all, it chooses two tours $k$ and $k^{\prime}$. Because of the fact that $k$ and $k^{\prime}$ might be suboptimal, they are pre-optimized by the routing heuristic 2-opt* [13]. 2-opt* solves a TSP by exchanging edges under consideration of time windows. Next, TS executes $\lambda$-interchanges as a neighborhood-structure on these tours, i.e. moves consisting of up to $\lambda_{\text {move }}$ unloading points and swaps consisting of up to $\lambda_{\text {swap }}$ unloading points with restriction to feasible solutions (capacity and time windows restriction) are operated (see [25]). This neighborhood-search can be subdivided into two types of exchanges:

- A move pushes up to $\lambda_{\text {move }}$ unloading points from tour $k$ to $k$ ' or from tour $k^{\prime}$ to tour $k$, respectively. The removed unloading points are inserted into the other tour by finding an insertion position with lowest costs. Hereby, the additional transport costs $t c_{a d d}$ of an unloading point $l$ are calculated for all possible insertion positions between unloading points $i$ and $j$ of the other tour:

$$
t c_{a d d}=t c_{i l}+t c_{l j}-t c_{i j}
$$

A move can also include a so called ejection chain, i.e. more than one unloading point. If an ejection chain (restricted to consecutive unloading points) has to be inserted in another tour (e.g. 3 unloading points $l, l^{\prime}$ and $l^{\prime \prime}$ are moved, see Figure 6 right hand side), the cheapest insertion position is found by minimizing

$$
t c_{a d d}=t c_{i l}+t c_{l^{\prime \prime} j}-t c_{i j}
$$

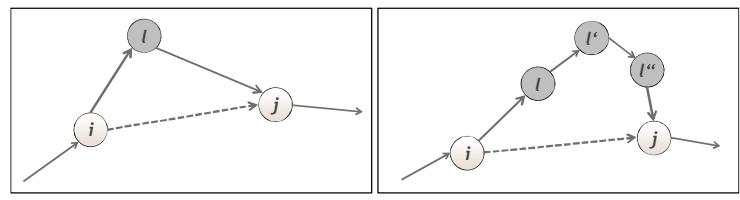

Figure 6. Computation of insertion position

Figure 7 contains two examples for moves: The left figure illustrates a $(1,0)$-move resulting in two new tours $[0,7,5,0]$ and $[0,2,3,1,4,6,0]$. The 
right figure shows a $(0,3)-$ move, i.e. three consecutive unloading points are pushed to tour 1. The resulting tours are $[0,1,3,4,6,7,5,0]$ and $[0,2,0]$. It might happen that a move results in a tour $[0,0]$, i.e. one vehicle can be saved.

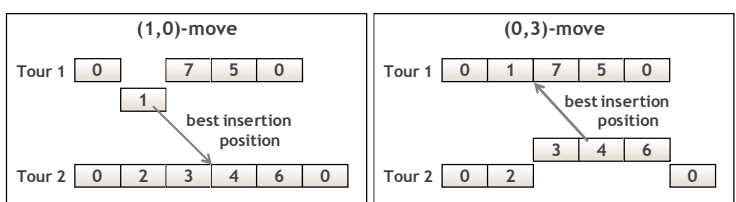

Figure 7. Move

Concerning moves, a neighborhood type of $(3,3)$ is used $\left(\lambda_{\text {move }}=3\right)$, i.e. the neighborhood types $(1,0),(2,0),(3,0)$ as well as $(0,1),(0,2)$ and $(0,3)$ are tested and evaluated. Moves with more than one unloading point are restricted to ejection chains.

- A swap exchanges up to $\lambda_{\text {swap }}$ unloading points from tour $k$ to $k^{\prime}$ and from tour $k^{\prime}$ to tour $k$. Hereby, all possible swaps are tested. The removed unloading points are inserted at the original position of the other tour's swapped unloading point(s). Figure 8 contains two examples for swaps: The left figure illustrates a $(1,1)$-swap resulting in two new tours $[0,1,7,3,0]$ and $[0,2,5,4,6,0]$. The right figure shows a $(1,2)-$ swap, i.e. one unloading point of tour 1 is swapped with two consecutive unloading points of tour 2 . The resulting tours are $[0,2,3,7,5,0]$ and $[0,1,4,6,0]$.

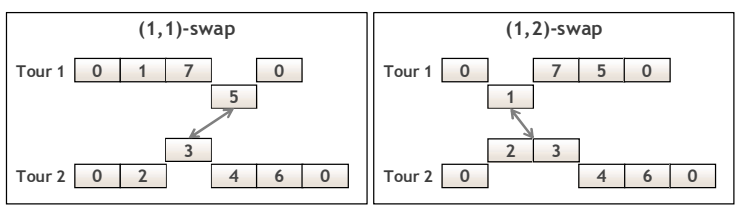

Figure 8. Swap

Concerning swaps, the neighborhood type $\left(\lambda_{\text {swap }}\right.$ $=2)$ is restricted to $(2,2)$, i.e. the swaps $(1,1)$, $(2,1),(1,2)$ and $(2,2)$ are executed in each iteration. In line with moves, swaps with more than one unloading point are restricted to ejection chains.

The best solution $s_{l}^{T S *} \in N\left(s_{l}^{T S}\right)$ generated through the neighborhood search initializes the next iteration even if it is worse than the current best solution. Solutions $s_{l}^{T S *}$ containing moves or swaps that are in the tabu list are forbidden unless a new very best solution is reached.
In order to communicate with the ants of the next ACO iteration $i t^{A C O}$, the pheromone trails are updated after completion of TS as proposed in the ACO II section.

\section{ACO II}

After $\sigma$ elitist ants constructed a TS-optimized set of solutions $\mathrm{S}^{*}$, pheromone trails are updated depending on the objective value of their solution. Hereby, the objective value of the $\mu$-th best elitist ant is defined as $L_{\mu}$. In some ant system approaches in literature all ants contribute to the trail update $[9,16]$. However, Bullnheimer et al. [8] suggest to rank the ants according to their quality of solution and to use only the best ranked ants (elitist ants) to update the pheromone trails. Hereby, the pheromone trail update for each $\operatorname{arc}(i, j)$ is defined as

$$
\tau_{i j}^{n e w}=\rho \tau_{i j}^{o l d}+\sum_{\mu=1}^{\sigma-1} \Delta \tau_{i j}^{\mu}+\sigma \Delta \tau_{i j}^{*}
$$

where $\rho$ is the trail persistence (with $0 \leq \rho \leq 1$ ). Thus, the trail is evaporated after each iteration by $(1-\rho)$. Only if an arc $(\mathrm{i}, \mathrm{j})$ was used by the $\mu$-th best ant, the pheromone trail is increased by $\Delta \tau_{i j}^{\mu}=\frac{(\sigma-\mu)}{L_{\mu}}$, and zero otherwise (second term in (13)). Additionally, all arcs belonging to the ever best solution (objective value $L^{*}$ ) are emphasized as if $\sigma$ elitist ants had used them. Thus, each elitist ant increases the trail intensity by an amount $\Delta \tau_{i j}^{*}$ that is equal to $\frac{1}{L^{*}}$ if arc $(\mathrm{i}, \mathrm{j})$ belongs to the ever best solution, and zero otherwise (cf. third term in (13)).

When $i t_{\max }^{A C O}$ iterations without a new optimum were executed, AntTabu finishes with the optimization of the ever best solution $L^{*}$. Hereby, each route of $L^{*}$ is post-optimized by the 2-opt*heuristic that exchanges arcs under consideration of the time window restriction.

\subsection{Software}

In order to manage the complex problem and its required data, a software including the algorithms 2opt* $^{*}$, ACO, TS and AntTabu was implemented in Java (see Figure 9). Moreover, the application comprehends a graphical user interface (GUI) and a multi-tier architecture (client/server) using Hibernate. The GUI allows the user to analyze production schedules including the allocation of editions to vehicles. In addition to that, vehicle routes can be illustrated by an integrated map and via a connection to Google Earth ${ }^{T M}$ or Microsoft MapPoint ${ }^{\circledR}$. Users have the possibility to calculate necessary distance 
and time matrices for the unloading points by means of MapPoint. Results can be automatically exported to Microsoft Excel ${ }^{\circledR}$.

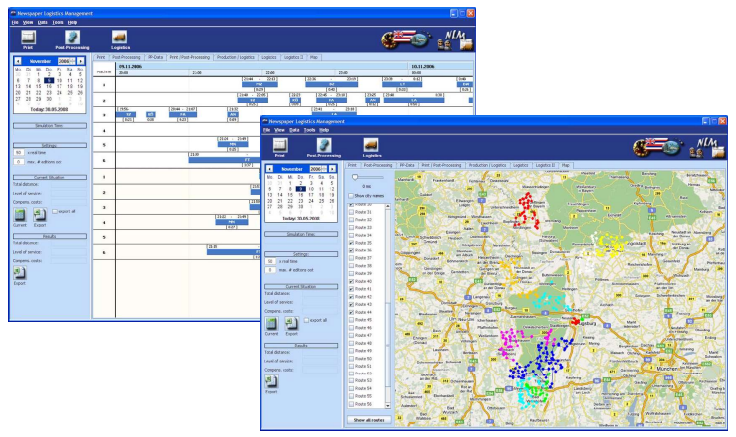

Figure 9. Software including AntTabu

\section{Application case}

AntTabu is exemplarily applied to one of the largest German newspaper companies (see section 4.1). Several different AntTabu parameters were tested to reach a high quality solution. The best settings are described in section 4.2. Finally, the results of AntTabu are compared to the current newspaper company's logistics costs (transport costs + newspaper carrier compensation costs).

\subsection{German newspaper company}

The main processes of this company can be described as follows. Each production day 19 different editions are produced on six parallel rotary print presses and six mechanical inserters located at one production site. After inserting pre-prints and advertising supplements the newspapers are allocated to currently 61 homogeneous vehicles executing tours with altogether 1425 determined unloading points to supply newspaper carriers (several newspaper carriers per unloading point distribute a total of 325,000 newspapers each day). All vehicles start their tours at the production site, delivering several different editions on about 300 days per year. Additionally, capacity restrictions of 2.8 tons per vehicle and one-sided time windows have to be kept. The company assesses variable delivery costs of $1 € /$ $\mathrm{km}$ and compensation costs of $0.20 €$ per minute of delay per newspaper carrier.

\subsection{Parameter settings}

AntTabu is applied to 44 randomly chosen production days. First of all different parameter settings in order to find the best values for the newspaper company were intensively evaluated. Therefore the total number of ants $(\varsigma)$, the number of elitist ants $(\sigma)$, the maximal number of iterations without a new best solution for ACO $\left(i_{\max }^{A C O}\right)$, the maximal number of iterations without a new best solution for TS $\left(i_{\text {max }}^{T S}\right)$, the evaporation factor $(\rho)$, the exploitation factor $\left(q_{0}\right)$, the parameters for relative influence of pheromone trails, visibility and tour start postponement $(\alpha, \beta, \gamma)$, the tabu list length, the maximal number of moved unloading points $\left(\lambda_{\text {move }}\right)$ and the maximal number of swapped unloading points $\left(\lambda_{\text {swap }}\right)$ were varied to find the best settings. After a large amount of tests (50 different settings with 10 runs each) it became obvious that the best solutions can be realized with the parameter settings $\varsigma=15, \quad \sigma=3, \quad i_{\max }^{A C O}=5, \quad i_{\max }^{T S}=30, \quad \rho=0.8, \quad q_{0}=0.95$, $\alpha=1.5, \beta=9.5, \gamma=3.0$, tabu list length $=15, \lambda_{\text {move }}=3$ and $\lambda_{\text {swap }}=2$. These settings showed the best results under consideration of the trade-off between computation time and quality of solution.

\subsection{Results}

The resulting costs of AntTabu concerning the randomly chosen 44 production days (20 runs each day) are compared with the current costs of the company. As the company uses static routes, the current transport costs add up to $375,790.80 €$ in total (regarding the 44 production days) and 8,540.70€ per day. Additionally, the current newspaper carrier compensation costs range between $46.60 €$ and $3,065.40 €$ on the 44 production days $(725.11 €$ on average). Figure 10 compares the current variable costs with the optimized variable costs (best achieved costs).

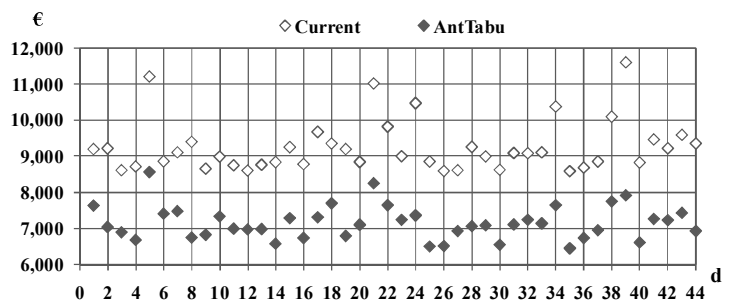

Figure 10. Result - current and optimized

\section{variable costs}

Summing up all optimized days the total variable costs can be decreased from currently 407,311.00€ to $314,442.50 €(-22.80 \%)$ with the best AntTabu results. Compared to the average variable costs of $322,209.10 €$, still a decrease of $20.89 \%$ can be realized. Since AntTabu contains stochastic elements, 
reliability and robustness are very important to assess the quality of optimization. Figure 11 shows that the different evaluation results are almost identical on each day. Only small deviations between best, average and worst objective values can be seen.

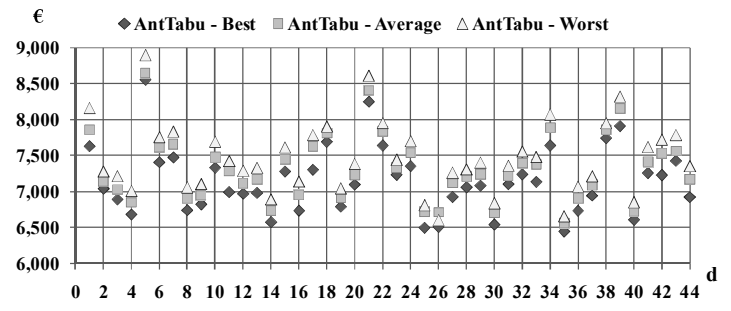

Figure 11. AntTabu - best/average/worst results

Having a look at the second part of the objective function, the carrier compensation costs can be eliminated on each optimized production day. This indicates that the AntTabu heuristic's clustering and routing procedure works so well, that all unloading points can be supplied in time. This leads to a level of service of $100 \%$ (customer satisfaction).

Although minimizing the number of vehicles is not an objective of the daily optimization in the newspaper industry (as the number of vehicles in a fleet is predetermined), another remarkable aspect is the reduction of vehicles on almost every day. Instead of currently 61 vehicles, an average of only 48.23 vehicles has to be used. Considering the best AntTabu results per day, the number of used vehicles ranges from 39 to 61 vehicles. Concerning the average results, it ranges from 40.25 to 64.68 (i.e. just on one optimized day additional vehicles would have to be used because of a significantly late production schedule).

Moreover, the claim for an efficient approach for the VRPTWCD is satisfied by AntTabu as computing times range between 30 and 45 minutes (Intel ${ }^{\circledR}$ Core $^{\text {TM }} 2$ CPU 6300 with 2 GB RAM).

\section{Conclusion}

This contribution presented a new kind of vehicle routing problem occurring especially in the newspaper industry. The well known VRPTW, which has been treated by many researches, was extended by cluster-dependent tour starts, resulting in a new vehicle routing problem (VRPTWCD). It introduces additional constraints that make the optimization even more difficult and implicates the need for a new solution procedure. As the most competitive approaches for a standard VRPTW - Ant Colony Optimization and Tabu Search - involve some disadvantages, these approaches were integrated and enhanced by additional parameters to face the VRPTWCD. The combination resulted in a new hybrid meta-heuristic as a first approach for the VRPTWCD - AntTabu - which is able to cluster and route simultaneously. This hybrid algorithm, included in an application software, was exemplarily applied to a real life application case of one of the largest newspaper companies in Germany (1425 unloading points are supplied each production day). As the results are very impressive, AntTabu proves to be adequate for the presented VRPTWCD in the newspaper industry: The overall variable costs were decreased by $22.80 \%$. Moreover, customer satisfaction was maximized and the number of used vehicles was lowered by $20.93 \%$ (on average).

Further research should consider the competitiveness of AntTabu with other solution methods presented in the literature. A comparison could be based on the widely used benchmark problems published by Solomon [30]. However, there are some problems to be solved: The Solomon instances are specified for a standard VRPTW, including equal tour start times per vehicle and twosided time windows (in contrast to the problem stated in this contribution). In addition to this, the benchmark problems do not include customer demands for multiple products (and thus clusterdependent tour starts). As AntTabu is designed for addressing a new problem that differs from the VRPTW in several aspects, a comparison with other heuristics is the main factor to be treated in further research.

\section{References}

[1] J.E. Beasley, "Route first - cluster second methods for vehicle routing", Omega, 11(4), Pergamon Press Ltd., Great Britain, 1983, pp. 403-408

[2] R. Bent and P. van Hentenryck, "A Two-Stage Hybrid Local Search for the Vehicle Routing Problem with Time Windows", Transportation Science, 38(4), INFORMS, Maryland, 2001, pp. 515-530.

[3] J. Berger, M. Barkaoui, and O. Bräysy, "A Routedirected Hybrid Genetic Approach for the Vehicle Routing Problem with Time Windows", INFOR, 41(2), Canada, 2001, pp. 179-194.

[4] O. Bräysy, "A Reactive Variable Neighborhood Search for the Vehicle Routing Problem with Time Windows", INFORMS J. on Computing, 15(4), INFORMS, Maryland, 2003, pp. 347-368.

[5] O. Bräysy, G. Hasle and W. Dullaert, "A multi-start local search algorithm for the vehicle routing problem with 
time windows", European Journal of Operational Research, 159(3), Elsevier, Holland, 2004, pp. 586-605.

[6] O. Bräysy and M. Gendreau, "Vehicle Routing Problem with Time Windows, Part I: Route Construction and Local Search Algorithms", Transportation Science, 39(1), INFORMS, Maryland, 2005, pp. 104-118.

[7] O. Bräysy and M. Gendreau, "Vehicle Routing Problem with Time Windows, Part II: Metaheuristics", Transportation Science, 39(1), INFORMS, Maryland, 2005, pp. 119-139.

[8] B. Bullnheimer, R.F. Hartl, and C. Strauss, "A new rank based version of the ant system: a computational study", Technical Report POM-03/97, Institute of Management Science, University of Vienna, Austria, 1997.

[9] B. Bullnheimer, R.F. Hartl, and C. Strauss, "Applying the Ant System to the Vehicle Routing Problem", Proceedings of the $2^{\text {nd }}$ International Conference on Metaheuristics MIC 97, France, 1997, pp. 1-12.

[10] B. Bullnheimer, G. Kotsis, and C. Strauss, „Parallelization Strategies for the Ant System“, Conference on High Performance Software for Nonlinear Optimization: Status and Perspectives, Ischia, 1997, Italy.

[11] B. Bullnheimer, R.F. Hartl, and C. Strauss, "An improved ant system algorithm for the vehicle routing problem", Annals of Operations Research, 89(0), Springer, Netherlands, 1999, pp. 319-328.

[12] J.F. Cordeau and G. Laporte, "Tabu Search Heuristics for the Vehicle Routing Problem", Technical Report G2002-15, Group for Research in Decision Analysis (GERAD), Montreal, 2002.

[13] G.A. Croes, "A method for solving traveling salesman problems", Operations Research, 6(6), INFORMS, Maryland, 1958, pp. 791-812.

[14] K. Doerner, M. Gronalt, R.F. Hartl, and M. Reimann, "SavingsAnts for the Vehicle Routing Problem", Proceedings of the Applications of Evolutionary Computing on Evoworkshops 2002, Springer, London, 2002, pp. 11-20.

[15] M. Dorigo, V. Maniezzo, and A. Colorni, "Positive feedback as a search strategy", Technical report 91-016, Dipartimento di Elettronica, Politecnico di Milano, Italy, 1991.

[16] M. Dorigo, V. Maniezzo, and A. Colorni, "Ant system: optimization by a colony of cooperating agents", IEEE Transactions on Systems, Man, Cybernetics - Part B, 26(1), New Hampshire, 1996, pp. 29-41.

[17] M. Dorigo and L.M. Gambardella, "Ant Colony System: A Cooperative Learning Approach to the Traveling Salesman Problem", IEEE Transactions on Evolutionary Computation, 1(1), UK, 1997, pp. 53-66.

[18] M. Dorigo and C. Blum, "Ant colony optimization theory: a survey", Theoretical Computer Science, 344(2-3), Elsevier Science Publishers Ltd., UK, 2005, pp. $243-278$.
[19] L.M. Gambardella, E. Taillard, and G. Agazzi, "MACS-VRPTW: A Multiple Ant Colony System for Vehicle Routing Problems with Time Windows", Technical Report IDSIA, Lugano, 1999.

[20] J.N. Holt and A.M. Watts, "Vehicle Routeing and Scheduling in the Newspaper Industry", In B.L. Golden and A.A. Assad (editors), "Vehicle Routing: Methods and Studies”, Amsterdam, 1988, pp. 347-358.

[21] J. Homberger and H. Gehring, "A two-phase hybrid metaheuristic for the vehicle routing problem with time windows", European Journal of Operational Research, 162(1), Elsevier, Amsterdam, 2005, pp. 220-238.

[22] T. Ibaraki, S. Imahori, M. Kubo, T. Masuda, T. Uno, and M. Yagiura, "Effective Local Search Algorithms for the Vehicle Routing Problem with General Time Window Constraints", Proceedings of the 4th Metaheuristics International Conference, 2001, pp. 293-297.

[23] R.J. Mantel and M. Fontein, "A practical solution to a newspaper distribution problem", International Journal of Production Economics, 30-31, Elsevier Ltd., 1993, pp. 591599.

[24] D. Mester and O. Bräysy, "Active guided evolution strategies for large-scale vehicle routing problems with time windows", Computers and Operations Research, 32(6), Elsevier Science Ltd. Oxford (UK), 2005, pp. 1593 1614

[25] I.H. Osman, "Metastrategy simulated annealing and tabu search algorithms for the vehicle routing problem", Annals of Operations Research, 41 (4), Springer Netherlands, 1993, pp. 421-451.

[26] M. Reimann, K. Doerner, and R.F. Hartl, "Analyzing a Unified Ant System for the VRP and Some of Its Variants", Lecture notes in computer science, 2611, Springer Berlin / Heidelberg, 2003, pp. 135-142.

[27] M. Reimann, K. Doerner, and R.F. Hartl, "D-Ants: Savings Based Ants divide and conquer the vehicle routing problem", Computer \& Operations Research, 31 (4), Elsevier Ltd., 2004, pp. 563-591.

[28] Y. Rochat and E. Taillard, "Probabilistic diversification and intensification in local search for vehicle routing", Journal of Heuristics, 1, Springer Netherlands, 1995, pp. 147-167.

[29] R. Russell, W.-C. Chiang, and D. Zepeda, "Integrating multi-product production and distribution in newspaper logistics", Computers \& Operations Research, 35(5), Elsevier Science Ltd. Oxford (UK), 2008, pp. 1576-1588.

[30] M. M. Solomon, "Algorithms for the Vehicle Routing and Scheduling Problems with Time Window Constraints", Operations Research, 35(2), INFORMS, Maryland, 1987, pp. 254-265.

[31] E. Taillard, "Parallel Iterative Search Methods for Vehicle Routing Problems", Networks, 23, 1993, pp. 661673. 